\title{
Lamin A/C mutation associated with lipodystrophy influences adipogenic differentiation of stem cells through interaction with Notch signaling
}

\begin{tabular}{|c|c|}
\hline Journal: & Biochemistry and Cell Biology \\
\hline Manuscript ID & bcb-2017-0210 \\
\hline Manuscript Type: & Article \\
\hline Date Submitted by the Author: & 05-Aug-2017 \\
\hline Complete List of Authors: & $\begin{array}{l}\text { Perepelina, Ksenia; Almazov National Medical Research Centre; Saint- } \\
\text { Petersburg State Univesity } \\
\text { Dmitrieva, Renata; Almazov National Medical Research Centre, Stem Cells } \\
\text { Ignatieva, Elena; Almazov National Medical Research Centre } \\
\text { Borodkina, Aleksandra; Institut citologii RAN } \\
\text { Kostareva, Anna; Almazov National Medical Research Centre; Sankt } \\
\text { Peterburgskij nacional'nyj issledovatel'skij universitet informacionnyh } \\
\text { tehnologij mehaniki i optiki } \\
\text { Malashicheva, Anna; Almazov National Medical Research Centre, Molecular } \\
\text { Cardiology; Saint-Petersburg State University, Faculty of Biology; Sankt } \\
\text { Peterburgskij nacional'nyj issledovatel'skij universitet informacionnyh } \\
\text { tehnologij mehaniki i optiki }\end{array}$ \\
\hline $\begin{array}{r}\text { Is the invited manuscript for } \\
\text { consideration in a Special } \\
\text { Issue? : }\end{array}$ & N/A \\
\hline Keyword: & lamin A, cell differentition, stem cells, Notch \\
\hline
\end{tabular}

\section{SCHOLARONE" $^{m}$ \\ Manuscripts}


1 Lamin A/C mutation associated with lipodystrophy influences adipogenic differentiation of

2 stem cells through interaction with Notch signaling

3

4 K. Perepelina ${ }^{1,2}$, R. Dmitrieva ${ }^{1}$, E. Ignatieva ${ }^{1}$, A. Borodkina $^{3}$, A. Kostareva $^{1,4}$,

5 A. Malashicheva ${ }^{1,2,4}$

6

7

81 Almazov National Medical Research Centre, Saint-Petersburg, Russia

92 Saint-Petersburg State University, Saint-Petersburg, Russia

103 Institute of Cytology, Russian Academy of Sciences, Saint-Petersburg, Russia

114 ITMO University, Institute of Translational Medicine, Saint-Petersburg, Russia.

15 Corresponding author: Anna Malashicheva

16 E-mail: malashicheva ab@alvazovcentre.ru

17 orcid.org/0000-0002-0820-2913

$18 \quad$ Tel./Fax 89217669143

19 
21 Lamin $\mathrm{A} / \mathrm{C}$ is involved in many cellular functions due to its ability to bind chromatin and 22 transcription factors and affect their properties. Mutations of $L M N A$ gene encoding lamin A/C 23 affect differentiation capacity of stem cells. However, signaling pathways involved in interaction 24 with lamins in cellular differentiation remain unclear. Lipodystrophy associated with $L M N A$ 25 mutation $\mathrm{R} 482 \mathrm{~L}$ causes loss of fat tissue. In this study we investigated the role of LMNA 26 mutation R482L in modulating Notch signaling activity in adipogenic differentiation of 27 mesenchymal stem cells. Notch was activated using lentiviral Notch intracellular domain. 28 Activation of Notch was estimated by expression of Notch-responsive genes by qPCR and by activation of luciferase CSL-reporter construct. Effect of LMNA mutation on Notch activation and adipogenic differentiation was analyzed in cells bearing lentiviral $L M N A W T$ or $L M N A$

31 R482L. We show that LMNA R482L contributes to down regulation of Notch activation in undifferentiated and differentiated cells and decreases adipogenic differentiation, when Notch is activated. Thus, lamin $\mathrm{A} / \mathrm{C}$ interacts with Notch signaling thereby influencing cellular differentiation and point mutation in $L M N A$ could halt this interaction. adipogenic differentiation 
INTRODUCTION

The nuclear lamina consists of intermediate filament proteins called lamins and primarily localized at the inner side of the nuclear envelope (Aebi et al. 1986). Most differentiated somatic cells express two A-type lamins, lamin A and lamin $\mathrm{C}$, which are alternative splice variants of one gene, LMNA (Lin and Worman 1993). The lamina has long been thought to ensure structural support for the nuclear envelope. The discoveries that different mutations in LMNA are associated with tissue-specific disorders has led to the suggestion that lamins have regulatory functions and are essential for maintaining the differentiated state of post-embryonic somatic tissues (Hutchison and Worman 2004).

How lamins regulate gene expression and cell differentiation remains largely unclear. Direct binding of lamins to DNA, chromatin, nucleosomes and histones has been shown in many studies, but the physiological relevance of these interactions is still unclear (Naetar et al. 2017). Long heterochromatic domains associated with lamins have been identified and named laminaassociated domains. A number of studies have shown that anchoring genes to the lamina correlates with tissue-specific gene repression leading to the concept that tethering of genomic regions to the lamina is required for stable repression of genes during differentiation (Amendola and van Steensel 2014; Braun and Barrales 2016). In particular, it has been shown that lamin $\mathrm{A} / \mathrm{C}$ could bind to specific promoter subregions that regulate gene expression during adipogenic differentiation (Lund et al. 2013). The underlying mechanism for how this interaction contributes to gene regulation is unknown.

9 One of the diseases caused by autosomal-dominant inherited mutations in A-type lamins is Dunnigan-type familial partial lipodystrophy (FPLD; OMIM \#151660) (Speckman et al. 2000). FPLD primarily affects adipocytes and is characterized by progressive loss of subcutaneous fat from upper and lower extremities (Hegele 2001). In FPLD, LMNA missense 
63

64

mutations substitute a highly conserved arginine at position 482, or lysine at position 486 in the C-terminal globular domain of lamin A/C (Shackleton et al. 2000). Several studies have shown that A-type lamins can influence adipogenic differentiation of stem cells (Boguslavsky et al. 2006; Lund et al. 2013; Malashicheva et al. 2015), but cellular pathways affected by lamin A interactions remain under investigated.

Lamin $\mathrm{A} / \mathrm{C}$ interacts with the nuclear envelope protein emerin, which is absent or mutated in X-linked Emery-Dreifuss muscular dystrophy (EDMD1) (Clements et al. 2000; Sakaki et al. 2001). Anchorage of emerin at the nuclear rim is dependent upon lamin A/C interaction (Sullivan et al. 1999). Failure to interact with lamin A in R482L mutated fibroblasts from a FPLD patient has been shown for emerin (Capanni et al. 2003). Emerin, in turn, is able to suppress Notch signaling (Lee et al. 2017). Involvement of lamins in regulating Notch has also been shown for progerin, a mutant form of lamin A, resulted from a deletion in LMNA gene, which causes premature-ageing disease Hutchinson-Gilford Progeria Syndrome (HGPS) (Scaffidi and Misteli 2008).

The focus of this study was to analyze lamin A/C interaction with Notch pathway during adipogenic differentiation. For this we studied the effect of the LMNA point mutation on Notch activity and adipogenic differentiation of mesenchymal stem cells. We used lentiviruses bearing either $L M N A W T$ encoding wild type lamin A/C or lentivirus bearing $L M N A R 482 L$ encoding mutant lamin $\mathrm{A} / \mathrm{C}$ associated with familial partial lipodystrophy. We show that $L M N A R 482 L$ contributes to down regulation of Notch activation in undifferentiated and differentiated stem cells and decreases adipogenic differentiation, when Notch is activated. 
87

88

89

90

91

92

93

94

\section{MATERIALS AND METHODS}

\section{Human samples}

The study was performed according to Helsinki declaration and approval was obtained from the local Ethics Committee of Almazov Federal Medical Research Centre. Written informed consent was obtained from all subjects prior tissue biopsy.

\section{Cell culture}

Human and rat cardiac mesenchymal stem cells (cMSC) from myocardial tissue were isolated, propagated and differentiated as described previously (Smits et al. 2009). Briefly, myocardial tissue was dissected onto small clamps and digested by collagenase II (Worthington) solution $(1 \mathrm{mg} / \mathrm{ml})$ for 2 hours at $37^{\circ} \mathrm{C}$. Then cell suspension was passed through the $40 \mathrm{mkm}$ strainer, centrifuged at $300 \mathrm{~g}$ for $5 \mathrm{~min}$ and cells were resuspended in growth medium $(22.5 \%$ EGM-2 (Stem Cell, USA), $67.5 \%$ M199, $10 \%$ fetal bovine serum (Hyclone), 1x nonessential amino, 50 units $/ \mathrm{mL}$ penicillin and $50 \mu \mathrm{g} / \mathrm{mL}$ streptomycin (Thermo Fisher Scientific), seeded on $0.1 \%$ gelatine-coated 96-well plate and clonally expanded. After expansion the cells were maintained in Petri dishes (Corning, USA) in growth medium, at $37^{\circ} \mathrm{C}$ and $5 \% \mathrm{CO}_{2}$. cMSC immunophenotype was verified with flow cytometer GuavaEasyCyte6 (Millipore, USA) using CD33, CD45, CD117, CD90, CD105, CD73, CD146, CD56 monoclonal antibodies (BD, USA) as previously described (Dmitrieva et al. 2015).

\section{Lentiviral constructs and transduction}

LMNA WT and LMNA R482L bearing lentiviral constructs were described previously (Malashicheva et al. 2015) as well as Notch-intracellular domain (NICD) bearing lentiviral construct (Kostina et al. 2016). Lentiviral packaging plasmids were a generous gift of D.Trono (École Polytechnique Fédérale de Lausanne, Switzerland); pLVTHM was modified by the addition of the T7 tag and chloramphenicol resistance gene $(\mathrm{cm})$, resulting in the pLVTHM-T7- 
111

112

113

$\mathrm{cm}$ vector. 12xCSL-luc construct was kind gift of prof. U. Lendahl (Karolinska Institute, Stockholm, Sweeden). The reporter sequence was subcloned into pLVTHM-T7-cm vector.

\section{Lentiviral production and transduction}

Lentiviral production was performed as described previously (Kostina et al. 2016). Briefly, 100-mm dishes of nonconfluent 293T cells were cotransfected with $15 \mu \mathrm{g}$ pLVTHM-T7NICD, $5.27 \mu \mathrm{g}$ pMD2.G, and $9.73 \mu \mathrm{g}$ packaging pCMV-dR8.74psPAX2 by calcium-phosphate method. The following day the medium was changed to fresh, and the cells were incubated for 24 hours to obtain high-titer virus production. Produced lentivirus was concentrated from supernatant by ultracentrifugation method at $20000 \mathrm{~g}$ for $2 \mathrm{~h}$, resuspended in $1 \% \mathrm{BSA} / \mathrm{PBS}$ and frozen in aliquots at $-80^{\circ} \mathrm{C}$. Concentrated viral particles were added one hour after cMSC trypsinisation; $6 \mathrm{~h}$ later the culture medium was changed.

Transgene expression from lentiviruses was verified as follows. For NICD-bearing virus transduction of cMSC was verified by westernblotting for NICD. For $L M N A$-bearing viruses rat cMSC were transduced with viruses bearing human LMNA and then immunocytochemical staining with antibodies against human lamin $\mathrm{A} / \mathrm{C}$ was done. The saturating efficiency of cMSC transduction was $40-60 \%$.

\section{Immunocytochemical staining}

After growing on cover slides, cells were fixed for 20 minutes in 1\% paraformaldehyde and permeablized in $1 \% \mathrm{BSA} / 0.1 \%$ Triton $\mathrm{X}-100 / \mathrm{PBS}$ for three minutes, followed by blocking in $1 \% \mathrm{BSA} / \mathrm{PBS}$ for one hour. Then cells were incubated for one hour with primary antibodies: against lamin A/C (Leica). Secondary antibodies conjugated with r Alexa488 (Invitrogen) were used. DAPI was used to visualize nuclei. Microphotographs were taken using AxioObserver Microscope (Zeiss) at x20 and x40 magnification with AxioVision software. 
135

136

137

138

139

\section{Differentiation efficiency and analysis of microphotographs}

Intact cells and cells transduced with virus carrying wild type LMNA WT or mutant LMNA cLMNA R482L were used in experiments. Adipogenic differentiation was induced in control and transduced cells by addition $0.5 \mathrm{mM}$ 3_isobutyl_1_methylxanthine, $1 \mu \mathrm{M}$ dexamethasone, and $1 \mu \mathrm{M}$ insulin to the standard culture medium. All experiments were performed with three biological replications, i.e. three independent experimental procedures. 14 days after the induction of differentiation the cells were stained with Oil Red O (Sigma, USA) to reveal fully differentiated cells. The images of cell differentiation were analyzed for the ratio of differentiated and undifferentiated cell areas with MosaiX software (Carl Zeiss Microsystems, Germany). Expression of adipogenic markers were estimated after 3 days of differentiation using qPCR.

\section{qPCR}

RNA from cultured cells was isolated using ExtractRNA (Eurogene, Russia). Total RNA (1 $\mu \mathrm{g})$ was reverse transcribed with MMLV RT kit (Eurogen, Russia). Real-time PCR was performed with $1 \mu \mathrm{L}$ cDNA and SYBRGreen PCRMastermix (Eurogen, Russia) in the Light Cycler system using specific forward and reverse primers for target genes. The thermocycling conditions were as follows: $95{ }^{\circ} \mathrm{C}$ for five minutes, followed by 45 cycles at $95{ }^{\circ} \mathrm{C}$ for 15 seconds and $60{ }^{\circ} \mathrm{C}$ for one minute. A final heating step of $65^{\circ} \mathrm{C}$ to $95{ }^{\circ} \mathrm{C}$ was performed to obtain melting curves of the final PCR products. Corresponding gene expression level was normalized to HPRT or GAPDH from the same samples. Changes in target genes expression levels were calculated as fold differences using the comparative $\Delta \Delta \mathrm{CT}$ method. Primers for human NOTCH1, HEY1, CCND3, CMYC, SLUG, PPARG, AP2 could be represented at request.

\section{Promoter activity assay}


159 In the construct the expression of the firefly luciferase gene is regulated by twelve CSL binding 160 sites upstream of a minimal TK promoter and the level of CSL promoter activity indicates the 161 transcriptional activation of Notch pathway. Cells were lysed using Luciferase Assay System 162 (Promega) according to the manufacturer recommendations $48 \mathrm{~h}$ after transduction. Luciferase 163 activity was measured with Synergy2 (BioTek, США). Samples were normalized by protein 164 content using Pierce BCA Protein Assay Kit (Thermo Scientific).

\section{Statistics}

166 Values are expressed as mean $\pm \mathrm{SD}$ of triplicate experiments. Groups were compared 167 using Student's t-test. A value of $\mathrm{P} \leq 0.05$ was considered significant. Statistical analysis was 168 performed by using R software (version 2.12.0; R Foundation for Statistical Computing, Vienna, 169 Austria). 
171

172

173

174

175

176

\section{RESULTS}

\section{$L M N A R 482 L$ causes disruption of lamin organization in cMSC}

We analyzed distribution of lamin $\mathrm{A} / \mathrm{C}$ in $\mathrm{cMSC}$ transduced with lentiviruses bearing wild type and mutant LMNA. For this we transduced rat cMSC with lentiviruses containing human sequences of LMNA WT and LMNA R482L, and then stained the cells with antibody specifically recognizing human lamin A/C (Fig.1). In control non-transduced cells and in the cells transduced with $L M N A$ WT nuclei show lamin A/C at the nuclear rim with no alterations in nuclear morphology. The cells transduced with $L M N A$ R482L show nuclear blebbing and alterations in nuclear morphology.

\section{$L M N A R 482 L$ causes down regulation of Notch target genes in cMSC}

All further experiments were done using human cMSC. To estimate interaction of lamin $\mathrm{A} / \mathrm{C}$ and Notch pathway we analyzed effect of mutant $L M N A$ on expression of Notch target genes in human cMSC. We transduced cMSC with lentiviruses bearing LMNA WT or LMNA $R 482 L$ and activated Notch signaling by transducing the cells with Notch-intracellular domain (NICD) and analyzed expression of HEY1, SLUG, CMYC (c-Myc), CCND3 (cyclin D3), genes known to be among main Notch transcriptional targets (Andersson et al. 2011). NICD substantially activated expression of HEY1 indicating activation of Notch signaling (Fig. 2). LMNA R482L caused statistically significant decrease in expression of $H E Y 1, S L U G, C M Y C$ (cMyc), CCND3 compared to $L M N A W T$. This data indicates that mutant lamin $\mathrm{A} / \mathrm{C}$ counteracts Notch signaling activation.

LMNA R482L impairs adipogenic differentiation of cMSC in the presence of Notch activation 
by addition of specific factors to growth medium. After 14 days the cells demonstrated strong adipogenic phenotype with accumulation of fat droplets revealed by Oil Red O staining (Fig. 3).

Adipogenic induction was also confirmed by early expression of specific adipogenic markers $A P 2$ and $P P A R G 3$ days after induction of adipogenic differentiation (Fig. 3).

To estimate interaction of lamin A/C and Notch pathway in adipogenic differentiation we analyzed effect of mutant $L M N A$ on expression of Notch target genes in differentiating cMSC. We transduced cMSC with lentiviruses bearing LMNA WT or LMNA R482L and activated Notch signaling by transducing the cells with Notch-intracellular domain (NICD) and induced adipogenic differentiation in control and transduced cells. Fig. 4 shows that Notch activation itself inhibited adipogenic differentiation, which was observed by counting the differentiated cell area and by expression of $A P 2$ and $P P A R G$ (Fig 4a, Fig. 4b). In the absence of Notch activation LMNA R482L did not have prominent effect on adipogenic differentiation of cMSC comparing to LMNA WT. In contrast, in the presence of Notch activation LMNA R482L caused statistically significant decrease in amount of differentiated cells as well as decrease in the expression of adipogenic markers $A P 2$ and $P P A R G$. Thus, we observed inhibiting action of $L M N A R 482 L$ on adipogenic differentiation of cMSC only in the presence of activated Notch signaling.

\section{LMNA R482L causes down regulation of Notch target genes in differentiated cMSC}

Next we sought to confirm contribution of Notch signaling to the observed influence of LMNA R482L on downregulation of adipogenic differentiation. We transduced cMSC with lentiviruses bearing LMNA WT or LMNA R482L and activated Notch signaling by transducing the cells with Notch-intracellular domain (NICD) and induced adipogenic differentiation of cMSC (Fig. 5). Expression of HEY1 and NOTCH1 decreased in the cells bearing LMNA R482L compared to LMNA WT, when Notch was activated. Correspondingly, luciferase activity of CSL reporter decreased in cMSC with LMNA R482L compared to LMNAWT. 
218

219

220

221

222

223

224

225

226

227

228

229

230

231

232

233

234

235

236

237

238

239

240

241

\section{DISCUSSION}

Our data show that LMNA R482L contributes to down regulation of Notch activation in undifferentiated and differentiated mesenchymal stem cells and decreases adipogenic differentiation, when Notch is activated, thus supporting the hypotesis that lamin A/C interacts with Notch pathway.

Mutations of $L M N A$ that cause laminopathies with various phenotypes affect diferent types of tissues such as muscular, fat or bone and reflect the ability of lamins $\mathrm{A} / \mathrm{C}$ to influence different types of cellular differentiaion (Malashicheva et al. 2015). Direct binding of lamins to different regions of chromatin in the course of cellular differentiation led to the concept that differentiation might be dependent on repression/activation of genes involved in this binding (Naetar et al. 2017), however specific pathways involved in these interactions are hardly known.

Structural anomalies of nuclear lamina caused by mutations in LMNA could contribute to altered interaction of lamins with chromatin. Lamin A/C fails to functionally assemble in fibroblasts bearing R482L derived from a FPLD patient (Capanni et al. 2003). In line with this in our experiments lentiviral LMNA R482L caused nuclear structural abnormalities seen as nuclear blebbing in cMSC.

Overexpression of lamin A has been reported to suppress adipogenic differentiation (Bermeo et al. 2015; Boguslavsky et al. 2006; Tilgner et al. 2009; Vadrot et al. 2014). Moreover it has recently been shown, that $\mathrm{R} 482 \mathrm{~W}$ mutation in $L M N A$ affects the regulatory activity of sterol response element binding protein 1 (SREBP1), a transcription factor that regulates hundreds of genes involved in lipid metabolism and adipocyte differentiation (Vadrot et al. 2014), thus proposing the mechanism for FLPD-associated LMNA mutation inhibition of adipogenesis. However, in our cellular model using mesenchymal stem cells of cardiac origin we did not observe the inhibition of adipogenic differentiation by $L M N A R 482 L$ alone comparing to 
$242 L M N A W T$, but only, when Notch was activated. This difference could be related to variations in 243 cellular context and origin.

244 Notch is one of the key pathways ensuring development, differentiation and maintenance 245 of adult tissues (Andersson et al. 2011). Notch is known to be involved in regulation of 246 adipogenic differentiation (Shan et al. 2016) and mainly inhibits adipogenic differentiation. 247 Implication of Notch in the interactions with lamins $\mathrm{A} / \mathrm{C}$ has been shown for progerin, a protein 248 resulted from a deletion of $L M N A$ gene and causing premature aging (Scaffidi and Misteli 2008).

249 Our data show that LMNA R482L causes down regulation of activated Notch and acts 250 synergistically with Notch in inhibiting adipogenic differentiation.

In a recent report emerin, lamin binding partner, has been shown to be able to directly

252 interact with Notch intracellular domain thereby suppressing Notch activity (Lee et al. 2017). According to another report emerin binding to R482L mutated lamin is impaired in the cells of FLPD patient (Capanni et al. 2003). Our findings on Notch downregulation by LMNA R482L in undifferentiated and differentiated cells are well in line with these reports. Interactions of lamin with Notch might be, thus, mediated by emerin.

In conclusion, our data suggest that lamin $\mathrm{A} / \mathrm{C}$ interacts with Notch signaling. Details and outcomes of this interaction deserve further research for better understanding the role of A-type lamins in cellular differentiation as well as the in pathology.

\section{Author contributions}

K.P. performed experiments, analyzed data, wrote manuscript, R.D., E.I., A.B., A.K. performed experiments, analyzed data, A.M. designed research, analyzed data, wrote the manuscript.

\section{Acknowledgments}

Work was funded by Russian Science Foundation under agreement 16-15-10178 
266

267

268

269

270

271

272

273

274

275

276

277

278

279

280

281

282

283

284

285

286

287

288

289

290

\section{REFERENCES}

Aebi, U., Cohn, J., Buhle, L., and Gerace, L. 1986. The nuclear lamina is a meshwork of intermediate-type filaments. Nature 323(6088): 560-564.

Amendola, M., and van Steensel, B. 2014. Mechanisms and dynamics of nuclear lamina-genome interactions. Curr. Opin. Cell Biol. 28: 61-68.

Andersson, E.R., Sandberg, R., and Lendahl, U. 2011. Notch signaling: simplicity in design, versatility in function. Development 138(17): 3593-3612.

Bermeo, S., Vidal, C., Zhou, H., and Duque, G. 2015. Lamin A/C Acts as an Essential Factor in Mesenchymal Stem Cell Differentiation Through the Regulation of the Dynamics of the Wnt/ $\beta \square$ Catenin Pathway. J. Cell. Biochem. 116(10): 2344-2353.

Boguslavsky, R.L., Stewart, C.L., and Worman, H.J. 2006. Nuclear lamin A inhibits adipocyte differentiation: implications for Dunnigan-type familial partial lipodystrophy. Hum. Mol. Genet. 15(4): 653-663.

Braun, S., and Barrales, R.R. 2016. Beyond Tethering and the LEM domain: MSCellaneous functions of the inner nuclear membrane Lem2. Nucleus 7(6): 523-531. doi:

10.1080/19491034.2016.1252892.

Capanni, C., Cenni, V., Mattioli, E., Sabatelli, P., Ognibene, A., Columbaro, M., Parnaik, V.K., Wehnert, M., Maraldi, N.M., Squarzoni, S., and Lattanzi, G. 2003. Failure of lamin A/C to functionally assemble in R482L mutated familial partial lipodystrophy fibroblasts: altered intermolecular interaction with emerin and implications for gene transcription. Exp. Cell Res. 291(1): 122-134. doi: https://doi.org/10.1016/S0014-4827(03)00395-1.

Clements, L., Manilal, S., Love, D., and Morris, G. 2000. Direct interaction between emerin and lamin A. Biochem. Biophys. Res. Commun. 267(3): 709-714.

Dmitrieva, R.I., Revittser, A.V., Klukina, M.A., Sviryaev, Y.V., Korostovtseva, L.S., Kostareva, A.A., Zaritskey, A.Y., and Shlyakhto, E.V. 2015. Functional properties of bone marrow derived 
291 multipotent mesenchymal stromal cells are altered in heart failure patients, and could be

292 corrected by adjustment of expansion strategies. Aging (Albany NY) 7(1): 14.

293 Hegele, R.A. 2001. Molecular basis of partial lipodystrophy and prospects for therapy. Trends

294 Mol. Med. 7(3): 121-126. doi: http://dx.doi.org/10.1016/S1471-4914(01)01930-X.

295 Hutchison, C.J., and Worman, H.J. 2004. A-type lamins: guardians of the soma? Nat. Cell Biol. 296 6(11): 1062-1067.

297 Kostina, A.S., Uspensky, V.E., Irtyuga, O.B., Ignatieva, E.V., Freylikhman, O., Gavriliuk, N.D., 298 Moiseeva, O.M., Zhuk, S., Tomilin, A., Kostareva, A.A., and Malashicheva, A.B. 2016. Notch299 dependent EMT is attenuated in patients with aortic aneurysm and bicuspid aortic valve.

300 Biochimica et Biophysica Acta (BBA) - Molecular Basis of Disease 1862(4): 733-740. doi:

301 http://dx.doi.org/10.1016/j.bbadis.2016.02.006.

302 Lee, B., Lee, T.-H., and Shim, J. 2017. Emerin suppresses Notch signaling by restricting the

303 Notch intracellular domain to the nuclear membrane. Biochimica et Biophysica Acta (BBA)-

304 Molecular Cell Research 1864(2): 303-313.

305 Lin, F., and Worman, H.J. 1993. Structural organization of the human gene encoding nuclear

306 lamin A and nuclear lamin C. J. Biol. Chem. 268(22): 16321-16326.

307 Lund, E., Oldenburg, A.R., Delbarre, E., Freberg, C.T., Duband-Goulet, I., Eskeland, R., 308 Buendia, B., and Collas, P. 2013. Lamin A/C-promoter interactions specify chromatin state309 dependent transcription outcomes. Genome Res. 23(10): 1580-1589.

310 Malashicheva, A., Bogdanova, M., Zabirnyk, A., Smolina, N., Ignatieva, E., Freilikhman, O., 311 Fedorov, A., Dmitrieva, R., Sjöberg, G., and Sejersen, T. 2015. Various lamin A/C mutations 312 alter expression profile of mesenchymal stem cells in mutation specific manner. Mol. Genet. 313 Metab. 115(2): 118-127.

314 Naetar, N., Ferraioli, S., and Foisner, R. 2017. Lamins in the nuclear interior- life outside the 315 lamina. J. Cell Sci. 130(13): 2087-2096. 
316

317

318

319

320

321

322

323

324

325

326

327

328

329

330

331

332

333

334

335

336

337

338

339

340

341

Sakaki, M., Koike, H., Takahashi, N., Sasagawa, N., Tomioka, S., Arahata, K., and Ishiura, S.

2001. Interaction between emerin and nuclear lamins. The Journal of Biochemistry 129(2): 321327.

Scaffidi, P., and Misteli, T. 2008. Lamin A-dependent misregulation of adult stem cells associated with accelerated ageing. Nat. Cell Biol. 10(4): 452-459.

Shackleton, S., Lloyd, D.J., Jackson, S.N., Evans, R., Niermeijer, M.F., Singh, B.M., Schmidt, H., Brabant, G., Kumar, S., and Durrington, P.N. 2000. LMNA, encoding lamin A/C, is mutated in partial lipodystrophy. Nat. Genet. 24(2): 153-156.

Shan, T., Liu, J., Wu, W., Xu, Z., and Wang, Y. 2016. Roles of Notch Signaling in Adipocyte Progenitor Cells and Mature Adipocytes. J. Cell. Physiol.

Smits, A.M., Van Vliet, P., Metz, C.H., Korfage, T., Sluijter, J.P., Doevendans, P.A., and Goumans, M.-J. 2009. Human cardiomyocyte progenitor cells differentiate into functional mature cardiomyocytes: an in vitro model for studying human cardiac physiology and pathophysiology. Nat. Protoc. 4(2): 232-243.

Speckman, R.A., Garg, A., Du, F., Bennett, L., Veile, R., Arioglu, E., Taylor, S.I., Lovett, M., and Bowcock, A.M. 2000. Mutational and haplotype analyses of families with familial partial lipodystrophy (Dunnigan variety) reveal recurrent missense mutations in the globular C-terminal domain of lamin A/C. Am J Hum Gen 66(4): 1192-1198.

Sullivan, T., Escalante-Alcalde, D., Bhatt, H., Anver, M., Bhat, N., Nagashima, K., Stewart, C.L., and Burke, B. 1999. Loss of A-type lamin expression compromises nuclear envelope integrity leading to muscular dystrophy. The Journal of cell biology 147(5): 913-920.

Tilgner, K., Wojciechowicz, K., Jahoda, C., Hutchison, C., and Markiewicz, E. 2009. Dynamic complexes of A-type lamins and emerin influence adipogenic capacity of the cell via nucleocytoplasmic distribution of $\beta$-catenin. J. Cell Sci. 122(3): 401-413.

Vadrot, N., Duband-Goulet, I., Cabet, E., Attanda, W., Barateau, A., Vicart, P., Gerbal, F., Briand, N., Vigouroux, C., and Oldenburg, A.R. 2014. The p. R482W substitution in A-type 
17

342 lamins deregulates SREBP1 activity in Dunnigan-type familial partial lipodystrophy. Hum. Mol.

343 Genet. 24(7): 2096-2109.

344

345

346

347

348

349

350

351 
352

353

354

355

356

357

358

359

360

361

362

363

364

365

366

367

368

369

370

371

372

373

FIGURE CAPTIONS

Figure 1

Verification of transgene $L M N A$ expression in cMSC. Rat cMSC were infected with saturating concentrations of lentiviruses bearing human LMNA WT or LMNA R482L genes. The cells were stained with anti lamin A antibody recognizing only human lamin A/C. Upper row: 20x magnification; lower raw 40x magnification. Human cMSC were used as a control (control) and give uniform nuclear staining of lamin $\mathrm{A} / \mathrm{C}$ at the nuclear rim. Transduction of rat $\mathrm{cMSC}$ with lentivirus bearing human LMNA WT shows nuclei with normal appearance and without any blebbing (LMNA WT). Transduction of rat cMSC with lentivirus bearing human LMNA R482L shows nuclear blebbing (LMNA WT) in some of the transduced cells as an effect of the mutation

Figure 2

LMNA R482L affects Notch target gene expression in cMSC. Notch was activated in cMSC by lentiviral transduction of Notch intracellular domain (NICD) and expression of Notch target genes was verified with the presence of either LMNAWT (WT) or LMNA R482L (R482L). The results are represented as mean $\pm \mathrm{SD} * \mathrm{p}<0.05$

Figure 3

cMSC are capable to adipogenic differentiation. Upper panel represents images of undifferentiated (left) and differentiated cells 14 days after induction of adipogenic differentiation with visible adipose droplets revealed by Oil red $\mathrm{O}$ staining. Lower panel represents analysis of adipogenic marker expression by qPCR in undifferentiated cells (Undiff) 
374

375

376

377

378

379

380

381

382

383

384

385

386

387

388

389

390

391

392 393

394

and after 14 days of adipogenic differentiation (Diff). The results are represented as mean $\pm \mathrm{SD}$;

$* * \mathrm{p}<0.05$

Figure 4

LMNA R482L affects differentiation ability of $\mathrm{cMSC}$, when Notch is activated. Adipogenic differentiation of cMSC was induced with/without Notch induction via transduction with lentivirus bearing Notch-intracellular domain (NICD) and in the presence of either LMNAWT (WT) or LMNA R482L (R482L). (A) Left panel: images of differentiated cells after Oil Red O staining. Right panel: results of differentiated cell area counts. The results are represented as mean \pm SD, $* \mathrm{p}<0.05$

Figure 5

LMNA R482L affects Notch target gene expression in differentiated cMSC. Adipogenic differentiation of cMSC was induced with/without Notch induction via transduction with lentivirus bearing Notch-intracellular domain (NICD) and in the presence of either LMNA WT (WT) or LMNA R482L (R482L. mRNA level of NOTCH1 and HEY1 was measured by qPCR. Notch-dependent transcription was measured as luciferase activity of CSL-luc reporter. The results are represented as mean $\pm \mathrm{SD}, * \mathrm{p}<0.05$ 

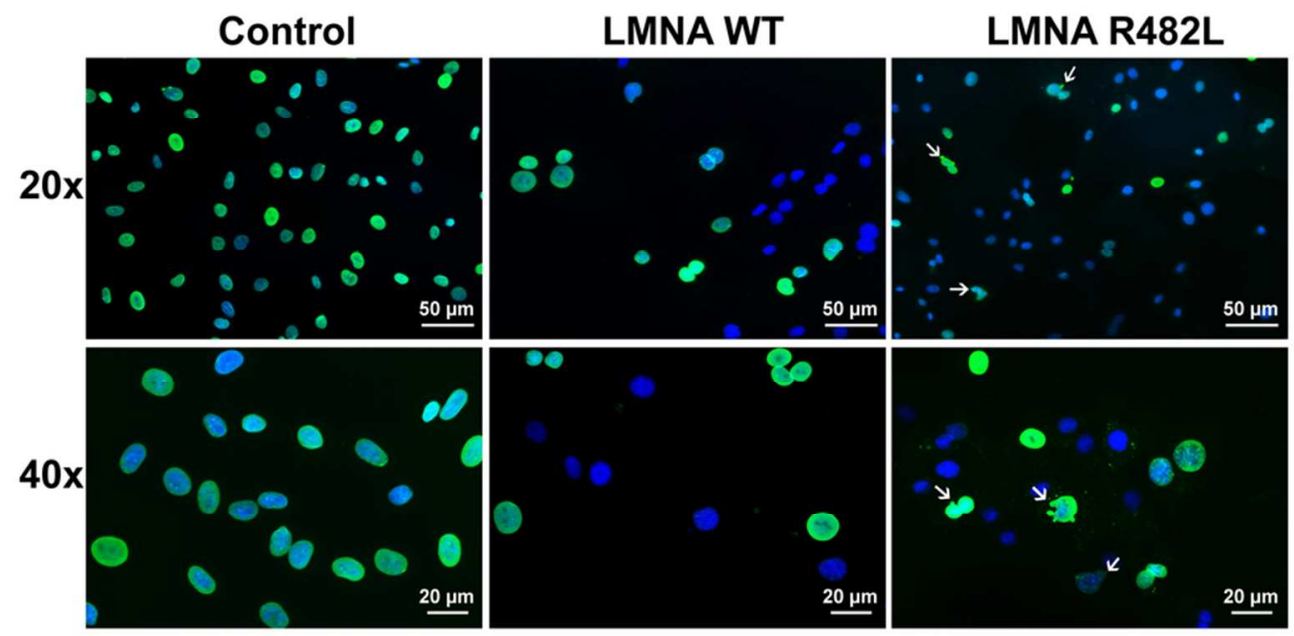

Figure 1

Verification of transgene LMNA expression in CMSC. Rat CMSC were infected with saturating concentrations of lentiviruses bearing human LMNA WT or LMNA R482L genes. The cells were stained with anti lamin A antibody recognizing only human lamin A/C. Upper row: 20x magnification; lower raw 40x magnification. Human cMSC were used as a control (control) and give uniform nuclear staining of lamin A/C at the nuclear rim. Transduction of rat CMSC with lentivirus bearing human LMNA WT shows nuclei with normal appearance and without any blebbing (LMNA WT). Transduction of rat CMSC with lentivirus bearing human LMNA R482L shows nuclear blebbing (LMNA WT) in some of the transduced cells as an effect of the mutation 

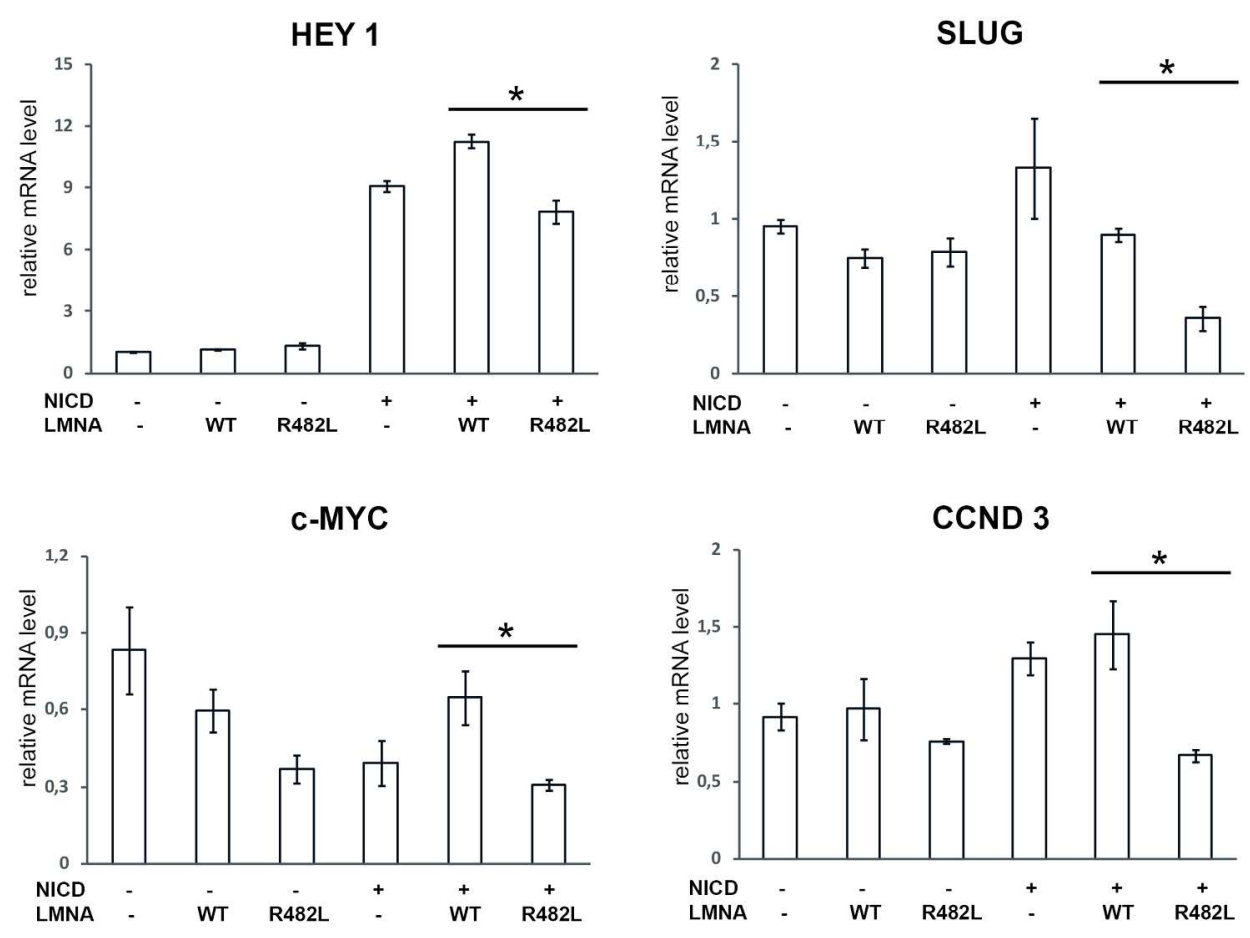

Figure 2

LMNA R482L affects Notch target gene expression in cMSC. Notch was activated in cMSC by lentiviral transduction of Notch intracellular domain (NICD) and expression of Notch target genes was verified with the presence of either LMNA WT (WT) or LMNA R482L (R482L). The results are represented as mean \pm SD

$* p<0.05$

$202 \times 149 \mathrm{~mm}(300 \times 300$ DPI $)$ 
Undiff. Diff.
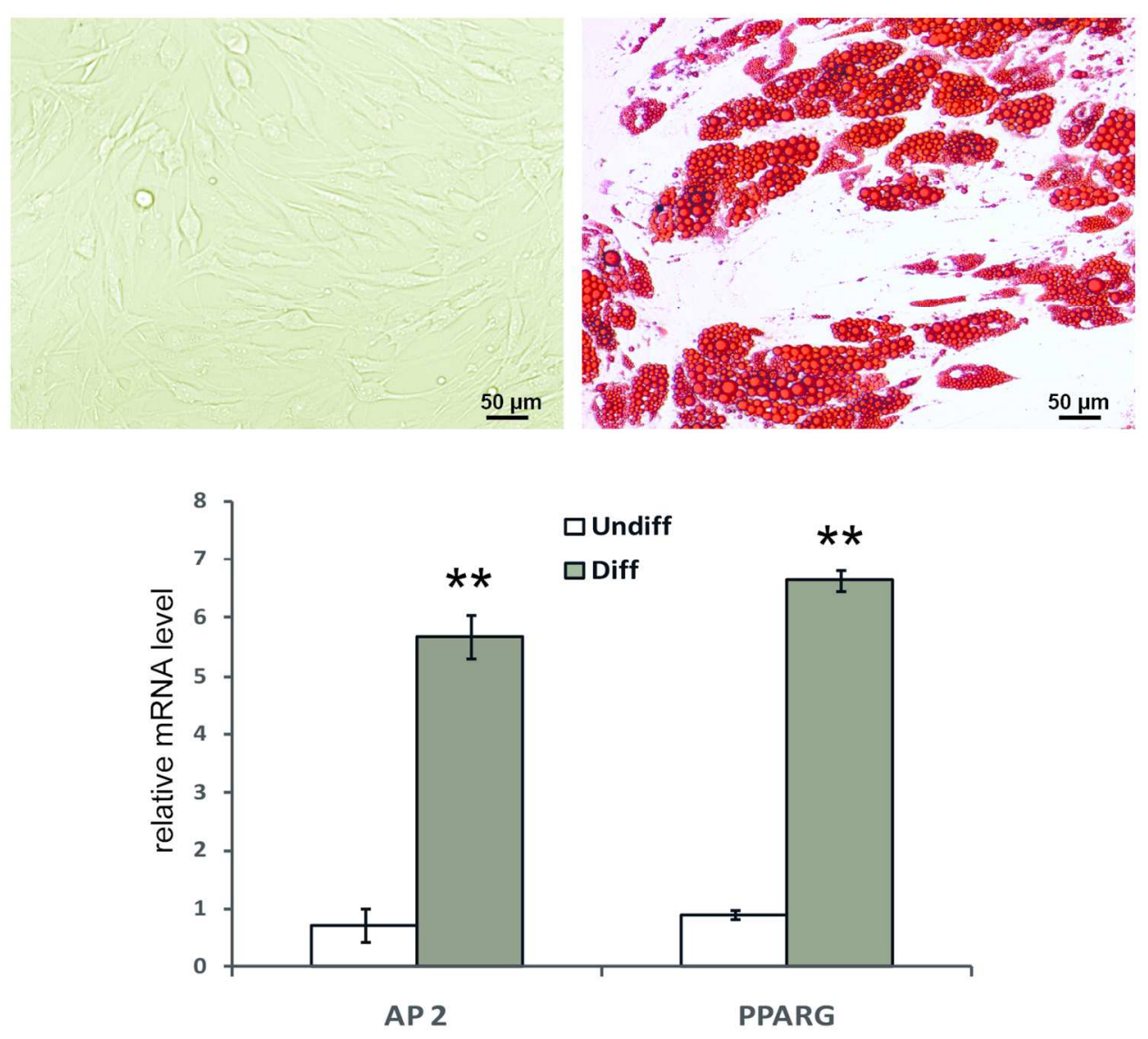

Figure 3

cMSC are capable to adipogenic differentiation. Upper panel represents images of undifferentiated (left) and differentiated cells 14 days after induction of adipogenic differentiation with visible adipose droplets revealed by Oil red $\mathrm{O}$ staining. Lower panel represents analysis of adipogenic marker expression by qPCR in undifferentiated cells (Undiff) and after 14 days of adipogenic differentiation (Diff). The results are represented as mean $\pm S D ; * * p<0.05$

$137 \times 131 \mathrm{~mm}(300 \times 300 \mathrm{DPI})$ 

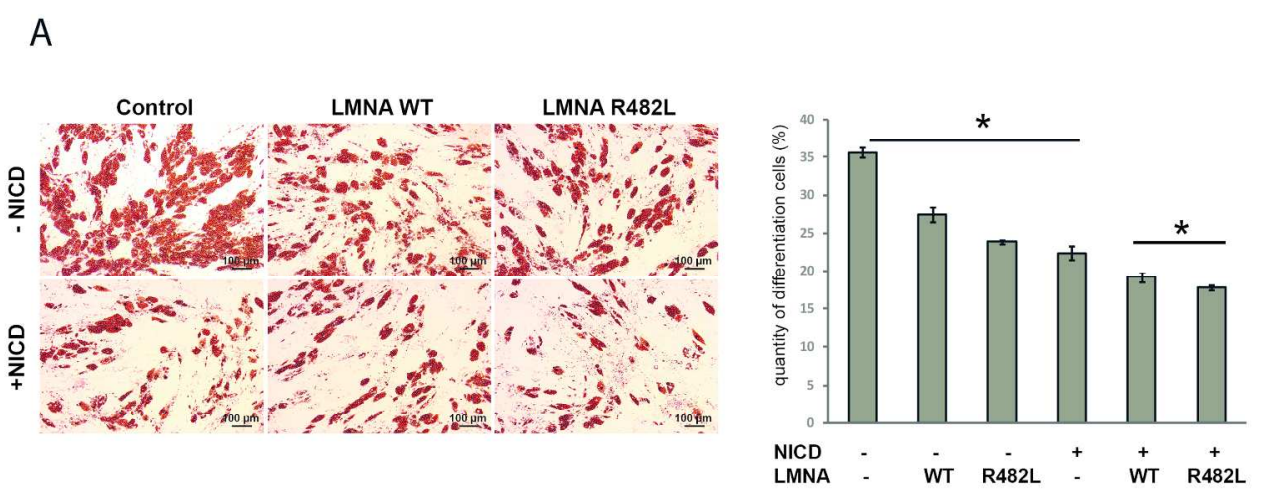

B
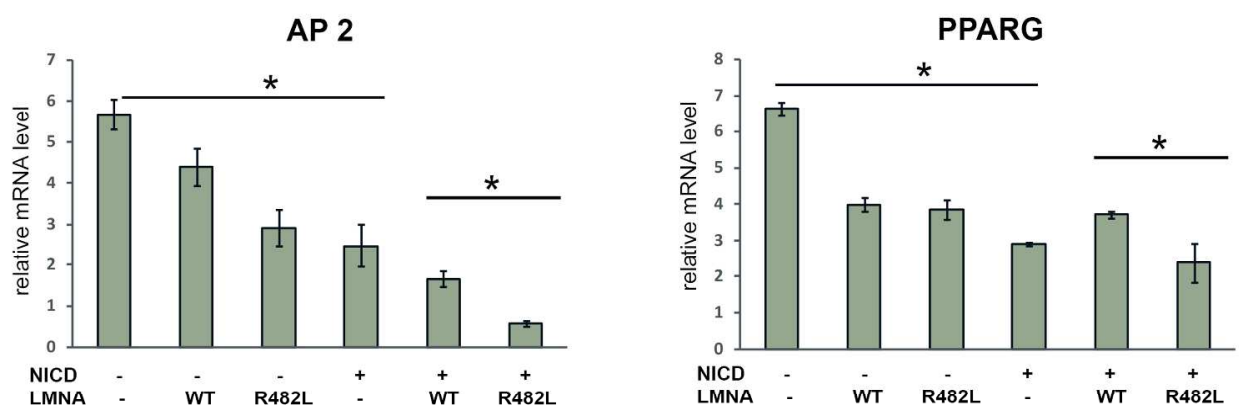

Figure 4

LMNA R482L affects differentiation ability of CMSC, when Notch is activated. Adipogenic differentiation of CMSC was induced with/without Notch induction via transduction with lentivirus bearing Notch-intracellular domain (NICD) and in the presence of either LMNA WT (WT) or LMNA R482L (R482L). (A) Left panel: images of differentiated cells after Oil Red $O$ staining. Right panel: results of differentiated cell area counts. The results are represented as mean $\pm \mathrm{SD},{ }^{*} \mathrm{p}<0.05$ 

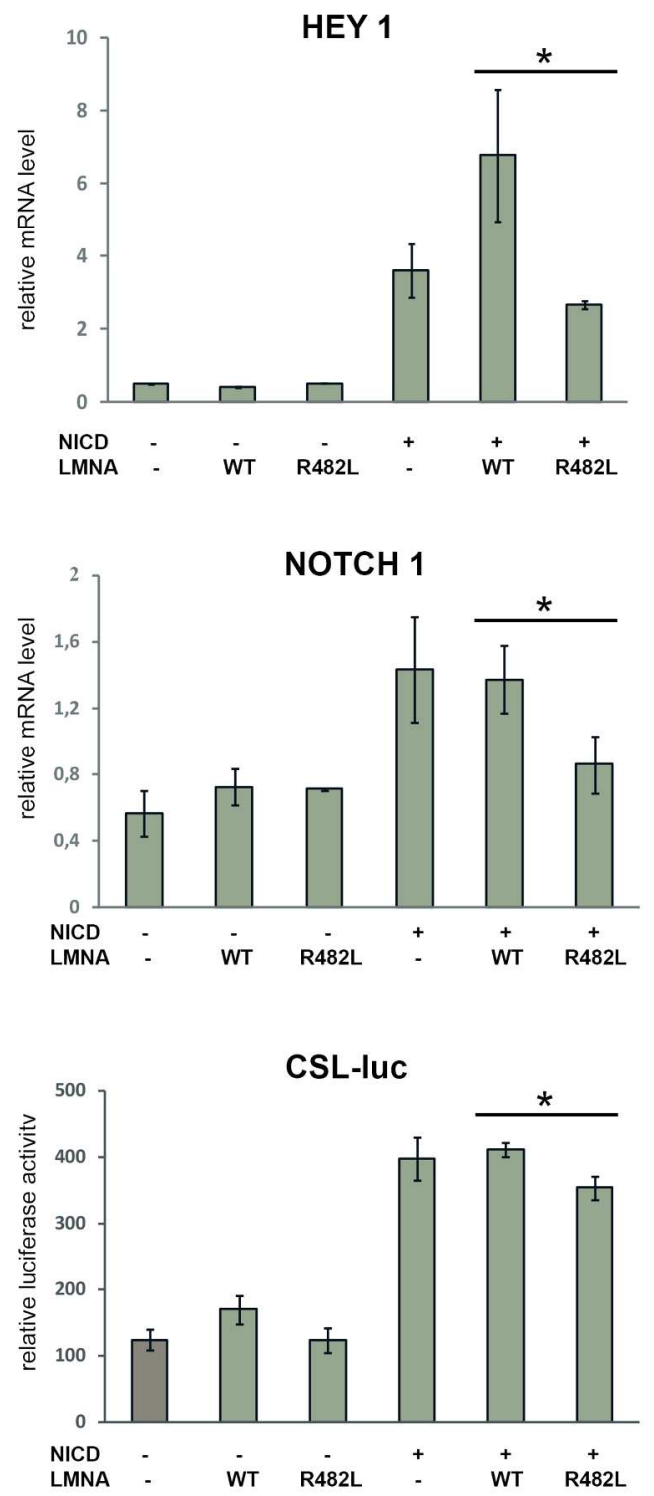

LMNA R482L affects Notch target gene expression in differentiated cMSC. Adipogenic differentiation of cMSC was induced with/without Notch induction via transduction with lentivirus bearing Notch-intracellular domain (NICD) and in the presence of either LMNA WT (WT) or LMNA R482L (R482L. mRNA level of NOTCH1 and HEY1 was measured by qPCR. Notch-dependent transcription was measured as luciferase activity of CSL-luc reporter. The results are represented as mean $\pm S D, * p<0.05$

$104 \times 237 \mathrm{~mm}(300 \times 300$ DPI $)$ 